\title{
Terpinolene, a component of herbal sage, downregulates AKT1 expression in K562 cells
}

\author{
NAOKO OKUMURA* , HITOMI YOSHIDA, YURI NISHIMURA, YASUKO KITAGISHI and SATORU MATSUDA* \\ Department of Environmental Health Science, Nara Women's University, Nara 630-8506, Japan
}

Received August 11, 2011; Accepted November 15, 2011

DOI: $10.3892 / \mathrm{ol} .2011 .491$

\begin{abstract}
Protein kinase AKT mediates cell proliferation and survival signals, and also contributes to cancer progression. Increased expression and/or activation of AKT is involved in a variety of human cancers. In cells treated with sage or rosemary extract, mRNA and protein expression levels of AKT1 were reduced compared with those of the control cells $48 \mathrm{~h}$ after the herbal treatments. We found that terpinolene, a common component of sage and rosemary, markedly reduced the protein expression of AKT1 in K562 cells and inhibited cell proliferation.
\end{abstract}

\section{Introduction}

The serine/threonine protein kinase AKT (also known as protein kinase B) mediates the cell survival signals coming through the phosphoinositide 3-kinase (PI3K) pathway by phosphorylation $(1,2)$. Human AKT has three isoforms: AKT1, AKT2 and AKT3. Once activated, AKT moves to the cytoplasm and nucleus, where it phosphorylates, activates or inhibits numerous downstream targets to regulate various cellular functions, including cell proliferation. Increased expression and activation of AKT is involved in a variety of human cancers. Although the activation of AKT alone is believed to be insufficient for tumorigenesis, it contributes to cancer progression by inhibiting apoptosis, promoting the cell proliferation and regulating their invasion abilities. Accordingly, AKT inhibitors are now in clinical development for the treatment of cancers (3).

Certain herbs are believed to possess numerous beneficial activities. The clinical use of herbal medications in therapy is widespread as herbs have mild bioavailability and also low toxicity (4). In one study, an inverse correlation was observed

Correspondence to: Professor S. Matsuda, Department of Environmental Health Science, Nara Women's University, Nara 630-8506, Japan

E-mail: smatsuda@cc.nara-wu.ac.jp

*Contributed equally

Key words: AKT, terpinolene, sage, rosemary, cancer, cell growth, apoptosis between the consumption of certain medicinal herbs, including sage and rosemary, and the incidence of lung cancer (5). In addition, traditional Chinese herbs have the characteristic of suppressing the expression of several genes involved in cancer (6). The inhibition of AKT expression is expected to interfere with the progression of cancer. We therefore hypothesized that certain medicinal herbs or spices affect the expression of AKT and therefore inhibit cell proliferation.

\section{Materials and methods}

Cell culture. The human cell lines K562, Daudi, Jurkat and U937 were maintained in RPMI-1640 supplemented with $10 \%$ fetal bovine serum (FBS), penicillin and streptomycin at $37^{\circ} \mathrm{C}$ in a humidified atmosphere containing $5 \% \mathrm{CO}_{2}$.

Preparation of extracts and reagents. Herb and spice powders were purchased at a food market in Japan. The powders were dissolved in $80 \%$ ethanol and subsequently diluted in $40 \%$ ethanol at a stock concentration of $50 \mathrm{mg} / \mathrm{ml}$. The mixtures were vortexed rigorously for $3 \mathrm{~min}$ followed by $3 \mathrm{~min}$ sonication. Following centrifugation at $1500 \mathrm{x}$ g for $5 \mathrm{~min}$, the supernatants were collected and stored at $-20^{\circ} \mathrm{C}$ until use. The reagents used were dissolved in ethanol and subsequently diluted at a stock concentration of $10 \mathrm{mM}$. The reagents were stored at $-20^{\circ} \mathrm{C}$ until use. For the cell treatments, a quantity of 0.5-10.0 $\mu \mathrm{l}$ was added into $1 \mathrm{ml}$ of cell culture medium.

Reverse transcriptase polymerase chain reaction ( $R T-P C R)$. Ubiquilin1, presenilin1 and GAPDH mRNAs were analyzed by semi-quantitative RT-PCR. Total RNA was extracted using an RNA isolation kit (Takara, Japan). Total RNA $(2 \mu \mathrm{g})$ was reverse transcribed using a Phusion RT-PCR kit (New England Biolabs, Ipswich, MA, USA) as described in the manufacturer's instructions. Cycle-based PCR was used to semi-quantitate the ubiquilin1 and presenilin1 gene level. GADPH was also used as an internal loading control. Samples were determined within 3 months of collection. The primers used for PCR were designed as follows: AKT1: forward, TCTATGGCGCTGAGATTGTG; and reverse, CTTAATGTGCCCGTCCTTGT (expected size, 116 bp); GAPDH: forward, TCCCATCACCATCTTCCA; and reverse, CATCACGCCACAGTTTCC (expected size, 376 bp). For real-time PCR, the reactions were performed in a real-time PCR system (Illumina, USA) using KAPA SYBR FAST reaction mix (Genetics, Japan). Thermocycling was performed 
according to the instructions at an annealing temperature of $60^{\circ} \mathrm{C}$ in a final volume of $10 \mu \mathrm{l}$ including Taq DNA polymerase.

Western blot analysis. An equal amount of protein samples were used for western blot analysis using anti-AKT1 (Cell Signaling Technology, Inc.), anti-Rb2 (BD Transduction Laboratories) and anti-Erk2 (AnaSpec, Inc., Fremont, CA, USA) antibodies, and quantified by densitometry. Western blots were repeated at least three times and the representative data were shown.

Cell proliferation assay. Cell proliferation activity was examined using Tetra Color One (Seikagaku Corporation, Japan). Cells were seeded onto 96 -well microplates (1,000 cells/well) and treated with extracts for 0, 24, 48, 72 and $96 \mathrm{~h}$. Following treatment, Tetra Color One was added according to the manufacturer's instructions. The optical density value of each well was measured using a microplate reader (BioRad iMark) with a test wavelength of $450 \mathrm{~nm}$.

Cytotoxicity assay. Cytotoxicity was examined using Annexin V-EGFP (Abcam) and 7-AAD Red (Enzo Life Sciences, Inc.). These detection reagents were used according to the manufacturer's instructions. Stained cells were detected by fluorescence microscopy (Eclipse Ti, Nikon).

\section{Results and Discussion}

Extracts of rosemary, green tea, sage, kuro-shitimi (red pepper), ginger, zingiber mioga or perilla frutescens were added into the cell culture media of the K562, Daudi, Jurkat or U937 cells, and the level of genes, including AKT1, was examined. RT-PCR analysis was employed to quantify the expression level of the gene. Total RNA was isolated $48 \mathrm{~h}$ after herbal extract treatment for the detection of AKT1, and the levels of mRNA were determined by conventional semi-quantitative RT-PCR (7). As shown in Fig. 1A, the AKT1 gene expression level was downregulated by the administration of sage or rosemary extracts at a final concentration of $50 \mu \mathrm{g} / \mathrm{ml}$, compared with the control ethanol vehicle. Similar results were obtained from the quantitative real-time RT-PCR analysis (Fig. 1B). No product was amplified in the no-template sample or when reverse transcriptions were omitted. However, the expression of anti-oncogenes $B R C A 1, B R C A 2, R b 1$ and $R b 2$ (data not shown) and the housekeeping gene GAPDH was unaltered (Fig. 1B). Differences among the gene expression profiles of K562, Daudi, Jurkat and U937 cells were insignificant (data not shown).

To examine the expression status at the protein level, Western blot analysis was used to analyze the AKT1 protein in the cells stimulated by sage or rosemary. As shown in Fig. 2, the sage or rosemary extracts markedly reduced the protein expression of AKT1, but not Erk2, in K562 cells, when cell cultures had been maintained in the herb stimulation for $48 \mathrm{~h}$. To rule out the possibility that sage or rosemary had any effects other than protein expression, we also checked several molecules relevant to cell proliferation. The expression of BRCA1, $B R C A 2, R b 1, R b 2$ and $E r k 2$ was then subjected to evaluation, since AKT1 affected the function of anti-oncogene products in cancer cells (8). We found that sage or rosemary extracts markedly enhanced the protein expression of $\mathrm{Rb} 2$ (Fig. 2, middle
$\mathbf{A}$
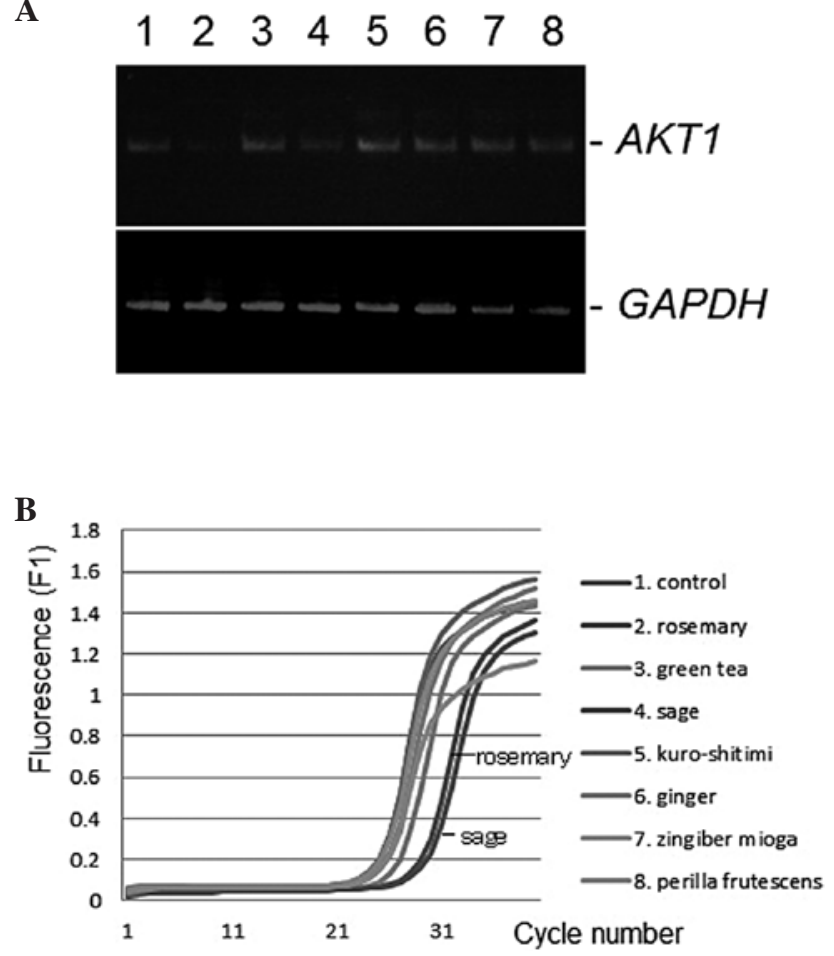

Figure 1. (A) Semi-quantitative RT-PCR was performed using primers specific to AKT1 or GAPDH on 100 ng total RNA prepared from K562 cells treated with rosemary, green tea, sage, kuro-shitimi (red pepper), ginger, zingiber mioga and perilla frutescens (lanes 2-8, respectively) or without any herb extracts (lane 1) at a final concentration of $50 \mu \mathrm{g} / \mathrm{ml}$ for $24 \mathrm{~h}$ The specific expression was determined in relation to the expression of the housekeeping gene GAPDH, which was used as an internal loading control. At least four independent experiments were carried out and typical paired results are documented. (B) Fluorescence data for PCR amplification plots of AKT1 in K562 cells stimulated by each of the indicated herbs or spices. RT-PCR was performed using primers specific to AKT1, as in Fig. 1A.

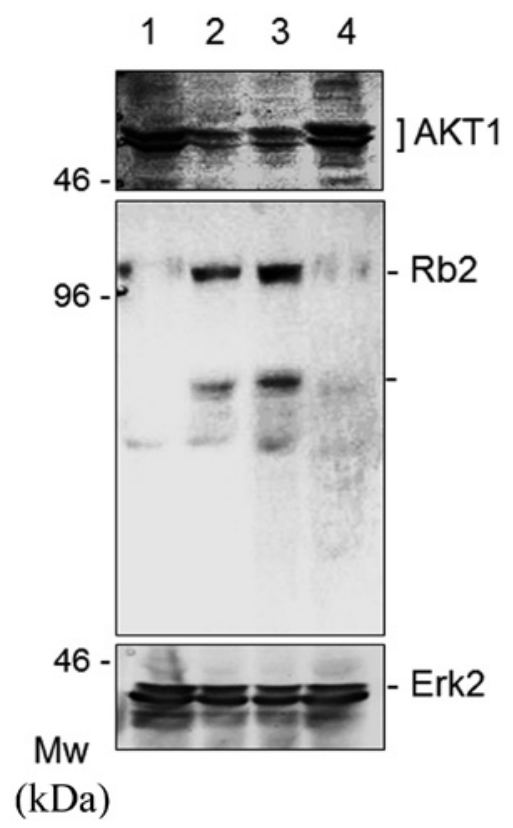

Figure 2. K562 cells were treated with rosemary, sage and green tea (lanes 2-4, respectively) or without any herb extracts (lane 1) at a final concentration of $50 \mu \mathrm{g} / \mathrm{ml}$ for $48 \mathrm{~h}$. Following treatment, cell lysates were isolated and the levels of AKT1 protein were detected by western blot analysis using anti-AKT1, anti-Rb2 and anti-Erk2 antibodies. At least three independent experiments were carried out and typical paired results are shown. 
A

122345678

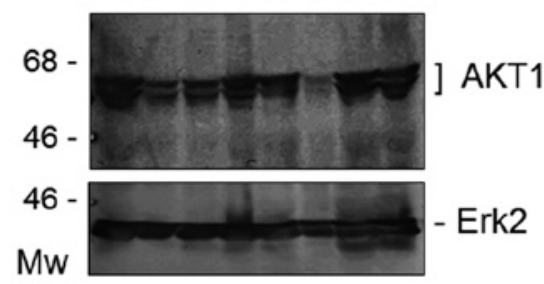

$(\mathrm{kDa})$

C<smiles>CC1=CCC(=C(C)C)CC1</smiles>

B

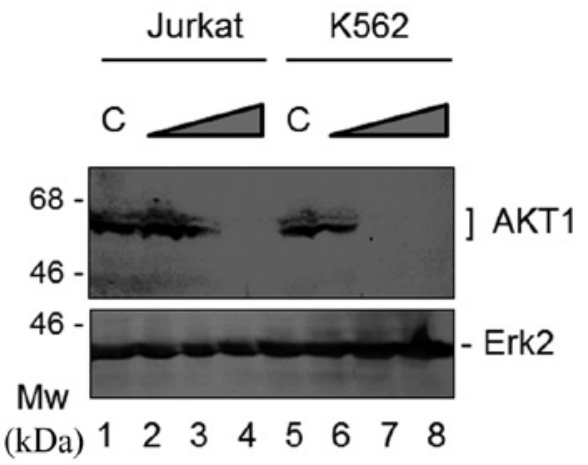

Figure 3. Terpinolene reduced the expression of AKT1. (A) K562 cells were treated with $50 \mu \mathrm{g} / \mathrm{ml}$ rosemary, $50 \mu \mathrm{g} / \mathrm{ml}$ sage, green tea, $0.05 \%$ linalool, $0.05 \%$ terpinolene, $0.05 \% \gamma$ terpinene and $0.2 \mathrm{mM}$ rosmarinic acid (lanes 2-6) with or without extracts of herbs (lane 1) for $48 \mathrm{~h}$. Following treatment, cell lysates were isolated and the levels of AKT1 and Erk2 protein were detected by western blot analysis using anti-AKT1 and anti-Erk2 antibodies. At least three independent experiments were carried out, and typical paired results are shown. (B) Dose-dependent inhibition of AKT1 protein expression by terpinolene. Jurkat and K562 cells were treated with or without (lanes 1 and 5) terpinolene at final concentrations of $0.001 \%$ (lanes 2 and 6), $0.01 \%$ (lanes 3 and 7 ) and $0.05 \%$ (lanes 4 and 8 ) for $48 \mathrm{~h}$. Protein levels were detected by western blot analysis using an anti-AKT1 antibody as in Figure 2. Western blotting with anti-Erk2 antibody also revealed equal levels of protein loading in each experiment. (C) Chemical structure of terpinolene.

A

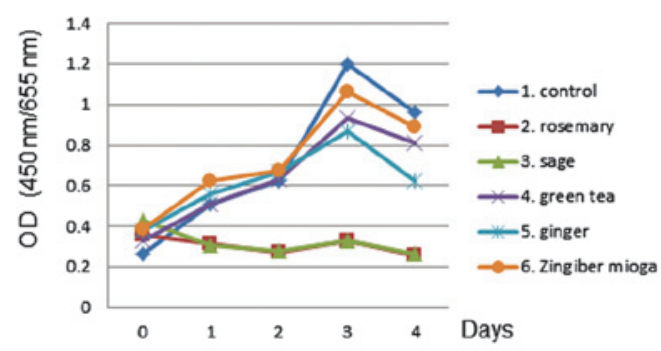

B

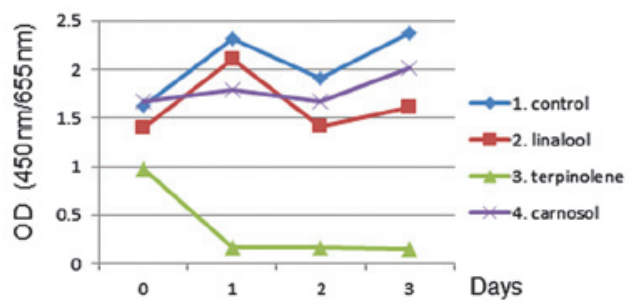

C

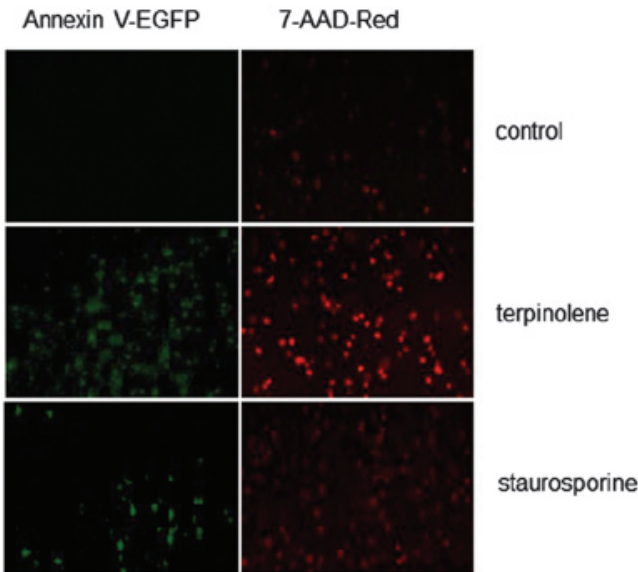

Figure 4. (A) Cell proliferation activity was examined using Tetra Color One. K562 cells were seeded onto 96-well microplates (1,000 cells/well) and treated with the indicated herbal extracts for $0,24,48,72$ and $96 \mathrm{~h}$. The optical density (OD) value of each well was measured using a microplate reader (BioRad). (B) K562 cells were seeded onto 96-well microplates (5,000 cells/well triplicate) and treated with a final concentration of $0.01 \%$ of the indicated reagents for 0 , 24,48 and $72 \mathrm{~h}$. These results were confirmed by more than three additional independent experiments. (C) Cytotoxicity was examined using Annexin V-EGFP (for apoptosis detection) and 7-AAD Red (for late apoptosis and necrosis detection). Stained cells were detected by fluorescence microscopy. Induction with $0.01 \%$ terpinolene or $1 \mu \mathrm{M}$ staurosporine was shown to yield distinct cell death.

panel). Similar results were also obtained with BRCA2 (data not shown).

Notably, sage and rosemary did not function synergistically to reduce AKT1 expression in K562 and other cells (data not shown). We addressed the question of whether a component of sage or rosemary was capable of reducing AKT1 expression. The common components of sage and rosemary are linalool, carnosol and terpinolene; these are 
expected to have anti-proliferative ability $(9,10)$. As shown in Fig. 3A, terpinolene markedly reduced the protein expression of AKT1; however, linalool or carnosol did not downregulate it. Following the treatment of the cells with various concentrations of terpinolene, western blot analysis revealed that the protein expression of AKT1, but not Erk2, was decreased with the increasing concentrations of terpinolene in the K562 and Jurkat cells (Fig. 3B). The final concentration of $0.05 \%$ terpinolene inhibited AKT1 expression by more than $95 \%$ in K562 and Jurkat cells (Fig. 3B). The chemical molecular structure of terpinolene is shown in Fig. 3C. It is produced by the alcoholic sulfuric acid treatment of pinene or by fractionation of turpentine.

The effect of sage or rosemary on cell proliferation activity was examined. The extracts were used in 3-day growth curves to determine the effects of cell proliferation. The assay revealed that cell proliferation activity was affected by sage or rosemary treatment in K562 cells, as shown in Fig. 4. Similar results were obtained with the use of Jurkat cells (data not shown). We again tested the effects of terpinolene on K562 cell proliferation activity and found that terpinolene significantly inhibited it (Fig. 4B). These results revealed the significant effect of terpinolene on K562 cell growth. We investigated whether terpinolene induced cytotoxicity in K562 cells. Results showed that terpinolene significantly induced cell apoptosis as much as staurosporine (Fig. 4C), unlike other molecules such as carnosol (data not shown).

The possibility of interactions between natural products and conventional prescription medicines is one of the most crucial issues in pharmacotherapeutic safety. Terpinolene is also used as a synthetic food flavoring additive or fragrance enhancer. Thus, it has high potential for human exposure. Although we have not yet identified the precise molecular mechanisms by which AKT1 expression is regulated, our findings provide new insight regarding cancer chemoprevention via the PI3K pathway and may help to establish strategies for a viable therapy.

\section{Acknowledgements}

This study was supported by Grants-in-Aid from the Ministry of Education, Culture, Sports, Science and Technology in Japan, and the Nara Women's University Intramural Grant for Project Research. In addition, this study was supported in part by a grant from the Fuji Foundation for Protein Research.

\section{References}

1. Castaneda CA, Cortes-Funes H, Gomez HL and Ciruelos EM: The phosphatidyl inositol 3-kinase/AKT signaling pathway in breast cancer. Cancer Metastasis Rev 29: 751-759, 2010.

2. Martelli AM, Evangelisti C, Chiarini F, et al: The emerging role of the phosphatidylinositol 3-kinase/Akt/mammalian target of rapamycin signaling network in normal myelopoiesis and leukemogenesis. Biochim Biophys Acta 1803: 991-1002, 2010.

3. Pal SK, Reckamp K, Yu H and Figurelin RA: Akt inhibitors in clinical development for the treatment of cancer. Expert Opin Investig Drugs 19: 1355-1366, 2010.

4. Abad MJ, Bedoya LM and Bermejo P: An update on drug interactions with the herbal medicine Ginkgo biloba. Curr Drug Metab 11: 171-181, 2010.

5. Johnson JJ: Carnosol: a promising anti-cancer and anti-inflammatory agent. Cancer Lett 305: 1-7, 2011.

6. Liu CP, Tsai WJ, Lin YL, Liao JF, Chen CF and Kuo YC: The extracts from Nelumbo Nucifera suppress cell cycle progression, cytokine genes expression, and cell proliferation in human peripheral blood mononuclear cells. Life Sci 75: 699-716, 2004.

7. Nishimura Y, Kitagishi Y, Yoshida H, Okumura N and Matsuda S: Ethanol extracts of black pepper or turmeric downregulated SIRT1 protein expression in Daudi culture cells. Mol Med Report 4: 727-730, 2011

8. Plo I, Laulier C, Gauthier L, Lebrun F, Calvo F and Lopez BS: AKT1 inhibits homologous recombination by inducing cytoplasmic retention of BRCA1 and RAD51. Cancer Res 68: 9404-9412, 2008.

9. Cherng JM, Shieh DE, Chiang W, Chang MY and Chiang LC: Chemopreventive effects of minor dietary constituents in common foods on human cancer cells. Biosci Biotechnol Biochem 71: 1500-1504, 2007.

10. Yoshida N, Takada T, Yamamura Y, Adachi I, Suzuki H and Kawakami J: Inhibitory effects of terpenoids on multidrug resistance-associated protein 2- and breast cancer resistance protein-mediated transport. Drug Metab Dispos 36: 1206-1211, 2008. 